\title{
Influence of sea ice lead-width distribution on turbulent heat transfer between the ocean and the atmosphere
}

\author{
S. Marcq and J. Weiss \\ Laboratoire de Glaciologie et de Géophysique de l'Environnement, UMR CNRS 5183, Université Joseph Fourier, \\ 54, rue Molière, BP 96, 38402 St Martin d'Hères cedex, France
}

Correspondence to: J. Weiss (weiss@lgge.obs.ujf-grenoble.fr)

Received: 12 September 2011 - Published in The Cryosphere Discuss.: 18 October 2011

Revised: 2 January 2012 - Accepted: 23 January 2012 - Published: 2 February 2012

\begin{abstract}
Leads are linear-like structures of open water within the sea ice cover that develop as the result of fracturing due to divergence or shear. Through leads, air and water come into contact and directly exchange latent and sensible heat through convective processes driven by the large temperature and moisture differences between them. In the central Arctic, leads only cover 1 to $2 \%$ of the ocean during winter, but account for more than $70 \%$ of the upward heat fluxes. Furthermore, narrow leads (several meters) are more than twice as efficient at transmitting turbulent heat than larger ones (several hundreds of meters). We show that lead widths are power law distributed, $P(X) \sim X^{-a}$ with $a>1$, down to very small spatial scales ( $20 \mathrm{~m}$ or below). This implies that the open water fraction is by far dominated by very small leads. Using two classical formulations, which provide first order turbulence closure for the fetch-dependence of heat fluxes, we find that the mean heat fluxes (sensible and latent) over open water are up to $55 \%$ larger when considering the lead-width distribution obtained from a SPOT satellite image of the ice cover, compared to the situation where the open water fraction constitutes one unique large lead and the rest of the area is covered by ice, as it is usually considered in climate models at the grid scale. This difference may be even larger if we assume that the power law scaling of lead widths extends down to smaller $(\sim 1 \mathrm{~m})$ scales. Such estimations may be a first step towards a subgrid scale parameterization of the spatial distribution of open water for heat fluxes calculations in ocean/sea ice coupled models.
\end{abstract}

\section{Introduction}

Sea ice is a fundamental component of the climate of polar regions. In the Arctic, the sea ice cover extends from about $5 \times 10^{6} \mathrm{~km}^{2}$ at the end of summer to $14 \times 10^{6} \mathrm{~km}^{2}$ in winter. Sea ice is not homogeneous and drifts, deforms and fractures as the result of external forcing (wind, ocean currents).
Leads develop as elongated cracks of open water in the ice cover due to divergence or shear in the sea ice motion, even in winter. Other processes, e.g. warm water upwelling at a particular location can also lead to an open water area within the ice pack, but we consider only fracture-induced leads in this study. Several studies have detected leads with remote sensing methods at a relatively large spatial resolution (Miles and Barry, 1998; Lindsay and Rothrock, 1995). In the Arctic, leads cover from $1-2 \%$ in the winter to $5-12 \%$ in summer of the total ice cover (Fichefet and Morales Maqueda, 1995). The positive trends in Arctic sea ice velocity and strain rates reported by Rampal et al. (2009), which related to increasing sea ice fracturing, may imply an increasing role of sea ice leads in heat transfer over the Arctic Ocean in the future.

Although the area of these openings is relatively small during winter, leads are of major importance for the heat balance. Through leads, air and water come into contact and directly exchange latent and sensible heat through convective processes driven by the large temperature difference between them (up to $30-40^{\circ} \mathrm{C}$ in winter). In summer, leads also play a large role in the absorption of shortwave radiation due to the low albedo of open water $(<0.1)$, compared to the albedo of multi year sea ice $(>0.60)$ (Fichefet and Morales Maqueda, 1995). The upward heat fluxes between open water and the atmosphere are orders of magnitudes larger than through thick ice: turbulent heat fluxes (sensible and latent ones) are less than $5 \mathrm{~W} \mathrm{~m}^{-2}$ over multi-year ice (Maykut, 1982) and can be up to $600 \mathrm{~W} \mathrm{~m}^{-2}$ over open water (Maykut, 1986; Andreas and Murphy, 1986).

Consequently, the variability of the sea ice fracturing could have a large impact on climate: as an example, for a sea ice cover with an open water fraction as little as $0.5 \%$, this fraction will contribute for about half of total thermal energy transfer between the ocean and the atmosphere (Heil and Hibler, 2002). In a modeling study, Lüpkes et al. (2008b) have found that a change of the lead fraction by $1 \%$ could cause a near-surface air temperature signal of up to $3.5 \mathrm{~K}$ 
under clear-sky conditions during polar night. They have also confirmed that the upward heat fluxes over leads are almost balanced by downward heat fluxes over the snow on nearby sea ice, as first shown by in situ data from the Surface Heat Budget of the Arctic Ocean (SHEBA) experiment (Overland et al., 2000). In spring, such warming of sea ice may induce a positive feedback by favoring surface melt and creating more openings, which in turn would release more heat in the atmosphere (Ledley, 1988).

Although climate modelers have been aware of the importance of leads in the heat balance of the polar regions, model parameterizations only take into account the sea ice concentration averaged at the grid scale and not the subgrid spatial distribution of open water. The surface in most models' grid boxes is considered to be the combination of two separated areas: one covered by ice and one covered by water. Heat fluxes are calculated separately over each area (Bitz et al., 2001; Gordon and O'Farrell, 2010; Goosse and Fichefet, 1999; Vavrus, 1995, etc.), using bulk formulae that are proportional to the temperature difference $\Delta T$ between the surface and the atmosphere at a certain height (typically $10 \mathrm{~m}$ ), to the wind speed $U$, and to a transfer coefficient for heat $C$, that usually depends on the stability of the atmosphere above and the roughness of the surface, but not on the lead-width distribution:

$$
H \sim C U \Delta T
$$

The formulation of $C$ relies on Monin-Obukhov similarity theory (Monin and Obukhov, 1954), but because there are several empirical formulations, different studies have slightly different coefficients and provide different results. Other studies chose to take into account the sea ice concentration in another way: Harvey (1988) has used a dependence of $C$ as a 2 nd order polynomial function of the sea ice concentration.

However, the actual lead distribution is very different from what is assumed in these models. The sea ice lead patterns are characterized by scale invariance (Weiss, 2003; Weiss and Marsan, 2004). As illustrated below, lead spacings and widths are power law distributed down to very small spatial scales, $P(X) \sim X^{-a}$ with $a>1$. This implies that the open water fraction is dominated by very small leads of the order of tens of meters or less, as the contribution of leads of width $\mathrm{X}$ scales as $X P(X) \sim X^{-(a-1)}$, i.e. strongly decreases with increasing width. At this scale, the discontinuity between water and ice leads to the creation of an atmospheric boundary layer whose depth and temperature depend heavily on the lead width (Venkatram, 1977; Stull, 1988). Alam and Curry (1997) and Andreas and Cash (1999) have proposed formulations of heat fluxes as functions of lead width based on several data sets.

Maslanik and Key (1995) calculated the sensitivity of turbulent flux estimates to changes in lead-width distribution using the heat flux parameterization of Andreas and Murphy (1986), and assuming that the distribution of leads followed an exponential distribution function $P(X)=\frac{1}{\lambda} \exp \left(-\frac{X}{\lambda}\right)$.
They argued that parameterizing these fluxes in a sea ice model can be done effectively using a single representative lead width (e.g. the mean lead width) rather than requiring a full distribution of lead widths. This is not surprising for an exponential distribution entirely defined by a single characteristic scale - the mean $\lambda$. As shown below, the situation is fundamentally different for a power law distribution.

In this study, we will use the formulations of Alam and Curry (1997) and Andreas and Cash (1999) and combine them with a distribution of lead widths in a real case study to estimate the heat fluxes at the scale of a model grid box $\left(\sim 60 \times 60 \mathrm{~km}^{2}\right)$. Then we will compare these heat fluxes to the bulk formulae used in large coupled models and GCMs. We will also analyze the sensitivity of the fluxes to the two parameters defining a power law distribution of lead widths, i.e. the exponent $a$ and the lower cut-off $L_{0}$.

\section{Lead-width dependence of turbulent heat fluxes}

\subsection{Physical process}

The sensible and latent heat fluxes occurring between sea ice and the atmosphere in the Arctic are largely dominated by the heat fluxes over open water: leads or polynyas. Through them, cold dry air and water directly exchange latent and sensible heat through convective processes. When air travels from a cooler (i.e. ice) to a warmer (i.e. water) surface, a convective atmospheric thermal internal boundary layer (TIBL) forms and deepens with distance downwind of the surface discontinuity or fetch $X_{\mathrm{f}}$ (Stull, 1988). Turbulence is vigorous in the TIBL and is driven by vertical wind shear (forced convection) and difference of buoyancy between warm air near the surface and cold air above it (free convection).

As the column of air advects over water, it gets warmer and more humid, hence the temperature and humidity differences between the air and the water surface lessen (Fig. 1). The sensible $\left(H_{\mathrm{s}}\right)$ and latent $\left(H_{1}\right)$ heat fluxes are proportional to the temperature and humidity differences, respectively, and thus decrease with increasing fetch.

\subsection{Analytical formulations of heat fluxes}

The dependence of $H_{\mathrm{S}}$ and $H_{1}$ to the fetch $X_{\mathrm{f}}$ is difficult to estimate and has been the focus of several studies (Alam and Curry, 1997; Andreas and Cash, 1999). Both studies provide first order turbulence closure.

The method of Andreas and Cash (1999) is mostly based on data fitting from different sets of measurements: the ALEX set (Andreas et al., 1979), which comes from measurements over natural and artificial leads with relatively short over-water fetch, the Smith et al. (1983) set, which comes from measurements over a semipermanent polynya in the Canadian Archipelago with larger fetch, and the Makshtas (1991) set, which comes from measurements over a refrozen polynya at drifting station North Pole 23. Note that 
the heat fluxes from the ALEX and the Makshtas (1991) sets are obtained by integrating the heat fluxes measured upwind and downwind of the lead, and the heat fluxes from the Smith et al. (1983) set are obtained by measurements at the downwind edge of the polynya.

The method of Alam and Curry (1997) is more of a theoretical approach based the Monin-Obukhov similarity theory (Monin and Obukhov, 1954) and the surface renewal theory as described by Brutsaert (1975), and uses the ALEX data to determine some empirical constants.

Andreas and Cash (1999) give direct formulations of the heat fluxes as a function of the lead width $X$. However, Alam and Curry (1997) give formulae for the heat fluxes as a function of fetch $X_{\mathrm{f}}$. In this section, note the indices $\mathrm{f}_{\mathrm{f}}$ that refer to formulae which are function of fetch $X_{\mathrm{f}}$. The sensible heat fluxes over a lead of width $X$ will then be calculated by integrating these fluxes from fetch 0 to fetch $X$, e.g.:

$H_{\mathrm{s}}(X)=\frac{1}{X} \int_{0}^{X} H_{\mathrm{s}_{\mathrm{f}}}\left(X_{\mathrm{f}}\right) d X_{\mathrm{f}}$

We first present the two studies briefly, and then use their results in the next section.

\subsubsection{The method of Andreas and Cash (1999)}

Andreas and Cash (1999) have developed an algorithm for computing sensible and latent heat transfer in fetch-limited conditions. They have used the heat flux definitions for free convection conditions that are valid for large fetch:

$H_{\mathrm{S}}(X)=C_{*} \rho c_{\mathrm{p}} D \frac{\Delta T}{\Delta z_{T}}$

$H_{1}(X)=C_{*} \rho L_{\mathrm{v}} D_{\mathrm{w}} \frac{\Delta Q}{\Delta z_{Q}}$

where $\Delta z_{T}$ and $\Delta z_{Q}$ are two length scales for heat and humidity, respectively, that take into account the viscosity of air and the buoyancy difference between the surface and the altitude $r$ (see Appendix A), $\rho$ is the air density, $c_{\mathrm{p}}$ is the specific heat of air at constant pressure, $L_{\mathrm{V}}$ is the latent heat of evaporation, $D$ and $D_{\mathrm{w}}$ are the molecular diffusivities of heat and water vapor in air, respectively, and $\Delta T=T_{s}-T_{r}$, $\Delta Q=Q_{s}-Q_{r}$, changes in heat and humidity, respectively between surface $s$ and altitude $r$. Note that the altitude $r$ corresponds to the first level of measurements in the atmosphere.

In the case of large fetch, the empirical non-dimensional coefficient $C_{*}$ is a constant, but the authors have determined $C_{*}$ as a function of stability, which allows them to generalize the fluxes formulae (3), (4) to the transition between free and forced convection, thus being useful for smaller fetch. Note that $C_{*}$ is used to calculate both sensible and latent heat fluxes. It is estimated with the lead and polynya data, and the best fit leads to:

$C_{*}=\frac{0.3}{0.4-h / L}+0.15$

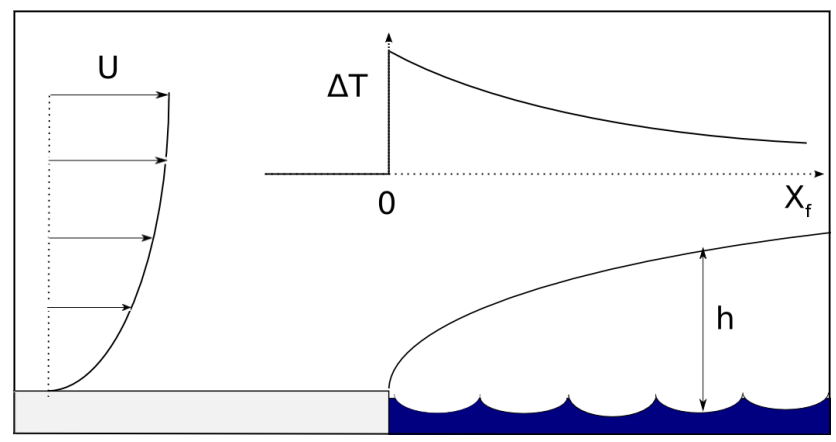

Fig. 1. Mean wind $U$ over sea ice, TIBL depth $h$ and temperature difference $\Delta T$ between water and the air in the TIBL as a function of fetch $X_{\mathrm{f}}$ after the discontinuity between ice and water.

where $h$ is the TIBL depth in meters as a function of lead width $X$ :

$h=0.82 \ln (X)+0.02$

and $L$ is the Obukhov length. $L$ is a length scale of stability, it is negative for unstable stratification and its magnitude increases with instability (see Appendix A). This formulation of the TIBL depth comes from lead and polynyas data (Fig. 1 of Andreas and Cash, 1999).

This method has been compared to the method developed in Andreas and Murphy (1986) and is valuable for wind speeds at height $2 \mathrm{~m}$ between 1 and $7 \mathrm{~m} \mathrm{~s}^{-1}$. The limit of validity of the approach of Andreas and Cash (1999) for small leads is difficult to estimate, as the key parameter used by these authors is $\frac{h}{L}$, not $X$. On Fig. 6 of Andreas and Cash (1999), Eq. (5) reasonably fits the data only for $\frac{h}{L}>0.2$. In our analysis below, $|L| \leq 1$, so - using Eq. (6) - the formulation is expected to be valid for lead widths $\mathrm{X}$ larger than several meters.

The main advantage of this method over the one described in Andreas and Murphy (1986) is that it is purely analytical and does not require iteration. However, the flux formulae depend heavily on the Eq. (5) for $C_{*}$ and Eq. (6) for $h$. These equations have been fitted on the data available (Figs. 1 and 6 of Andreas and Cash, 1999). A large part of the variability of these data is not explained by these fits. One explanation may be that Eq. (6) only depends on the lead width $\mathrm{X}$ and not on external conditions. However, Lüpkes et al. (2008a) showed that the TIBL depends on the boundary layer wind speed, the surface buoyancy over the lead and on the background mixed layer height for near-neutral inflow. It would be interesting to have more measurements taken for different external conditions to better constrain these formulae.

\subsubsection{The method of Alam and Curry (1997)}

Alam and Curry (1997) have developed an algorithm which uses the turbulent flux model described by Clayson et al. (1996) - based on the Monin-Obukhov similarity theory and 


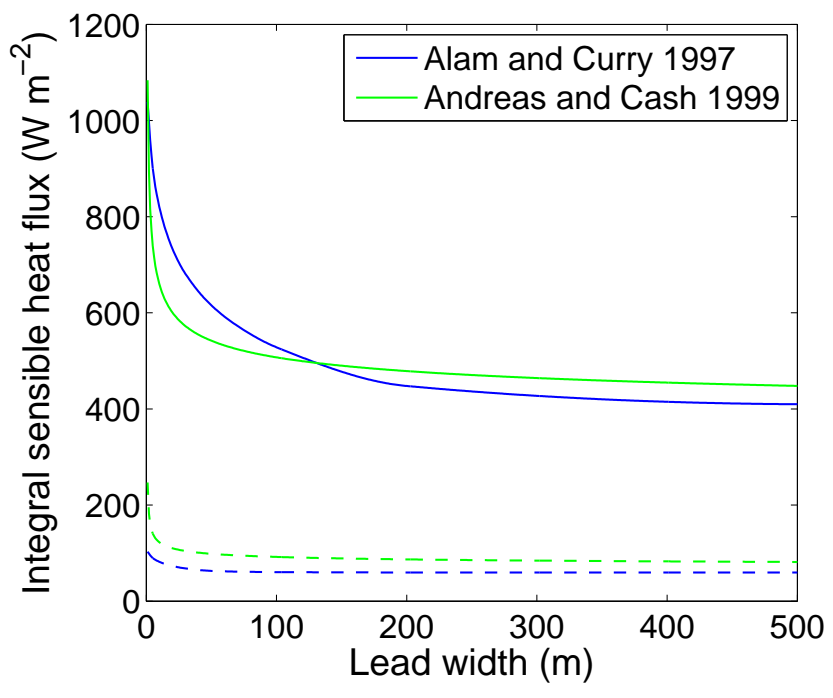

Fig. 2. Integral sensible heat flux as function of lead width computed using the methods of Alam and Curry (1997) - blue - and of Andreas and Cash (1999) - green. Solid and dashed lines correspond to $\Delta T=30^{\circ} \mathrm{C}, U=7 \mathrm{~m} \mathrm{~s}^{-1}$ and $\Delta T=10^{\circ} \mathrm{C}, U=3 \mathrm{~m} \mathrm{~s}^{-1}$, respectively.

on the surface renewal theory described by Brutsaert (1975) and a surface roughness model based on the model described by Bourassa et al. (2001). The turbulent flux of sensible heat is calculated as:

$H_{S_{\mathrm{f}}}=-\rho c_{\mathrm{p}} u_{*} T_{*}$

$T_{*}$ and $u_{*}$ are the Monin-Obukhov similarity scaling parameters for temperature and horizontal wind speed that correspond to the following empirical profiles for velocity and temperature in the surface layer:

$$
\begin{aligned}
& \frac{T_{r}-T_{s}}{T_{*}}=\frac{P r_{t}}{k}\left(\ln \left(\frac{r}{z_{0_{T}}}\right)-\Psi_{T}\right) \\
& \frac{u_{r}-u_{s}}{u_{*}}=\frac{1}{k}\left(\ln \left(\frac{r}{z_{0}}\right)-\Psi_{u}\right)
\end{aligned}
$$

where $T_{r}$ and $u_{r}$ (resp. $T_{s}$ and $u_{s}$ ) are the temperature and wind speed at altitude $r$ (resp. at the surface $s$ ), $P r_{\mathrm{t}}=0.85$ is the turbulent Prandtl number, $k=0.4$ is the Von Karman constant, $z_{0}$ and $z_{0_{T}}$ are the surface roughness lengths for momentum and heat, respectively, and $\Psi_{u}$ and $\Psi_{T}$ the respective stability functions, which are non-dimensional functions of $r / L$. These functions follow the formulation of Benoit (1977) for unstable conditions.

The surface roughness length for momentum $z_{0}$ is calculated using a model based on the model described by Bourassa et al. (2001). It takes into account the state of the water surface and particularly the nature of the waves at the surface (smooth surface, capillary waves or gravity waves). The surface roughness length for sensible heat $z_{0_{T}}$ uses the surface renewal theory (Brutsaert, 1975; Clayson et al., 1996). Note that the surface roughness parameterizations assume that the surface is open water. However, at very low air temperatures $\left(-20\right.$ to $\left.-30^{\circ} \mathrm{C}\right)$, open water in leads will be covered very quickly by a thin layer of ice (Pinto et al., 2003) causing a change in surface roughness. This is a source of uncertainty in this model.

The fit on the ALEX data is done through the introduction of the coefficient $c_{\text {conv }}$ which influences the surface renewal timescale and therefore the surface roughness length for sensible heat $z_{0_{T}}$ :

$c_{\mathrm{conv}}=\min \left[11\left(\frac{X_{\mathrm{f}} g}{u_{r}^{2}}\right)^{0.8}, 200\right]$

where $g$ is the acceleration due to gravity.

The two numbers 11 and 200 are empirical and determined to fit the ALEX data as well as possible. Alam and Curry (1997) have proposed calculations of sensible heat fluxes down to $1 \mathrm{~m}$-wide leads.

This method is complex and involves numerous parameters. We advise the reader to consult the original study: Alam and Curry (1997). Their Table 2 gives the lead integral sensible heat fluxes as function of lead width for the background atmospheric conditions that we have considered.

\subsection{Comparison of the two methods}

The methods of Alam and Curry (1997) and of Andreas and Cash (1999) give heat fluxes of similar magnitude but they have a different dependence upon lead width. Figure 2 shows the sensible heat fluxes computed by both methods in case of low wind speed and high atmospheric temperature (dashed lines: small fluxes) and in case of high wind and low atmospheric temperature (solid lines: large fluxes). For both methods, fluxes decrease strongly with increasing lead width (from $1000-1100 \mathrm{~W} \mathrm{~m}^{-2}$ to $500-550 \mathrm{~W} \mathrm{~m}^{-2}$ for large fluxes and from $100-250 \mathrm{~W} \mathrm{~m}^{-2}$ to $60-80 \mathrm{~W} \mathrm{~m}^{-2}$ for small fluxes). However, the method of Andreas and Cash (1999) has a much stronger dependence on lead width with very steep variations in heat flux for narrow leads $(1-25 \mathrm{~m})$. We'll see in Sect. 3 that the lead-width distribution follows a power law $P(X) \sim X^{-a}$, therefore small leads are by far the most numerous and we expect the sensible heat fluxes calculated with this method to have an even stronger dependence on the distribution of leads.

However, this method is less sensitive to the change in background atmospheric conditions than the method of Alam and Curry (1997): heat fluxes calculated using the study of Alam and Curry (1997) are equal to only $40-70 \%$ of the fluxes calculated using the study of Andreas and Cash (1999) for small fluxes but are relatively larger (90-120\%) for large fluxes.

Although Alam and Curry (1997) have used a parameterization via $c_{\text {conv }}$ that affects indirectly $T_{*}$ and thus $H_{\mathrm{f}}$ to fit to the same data that Andreas and Cash (1999) followed, the 


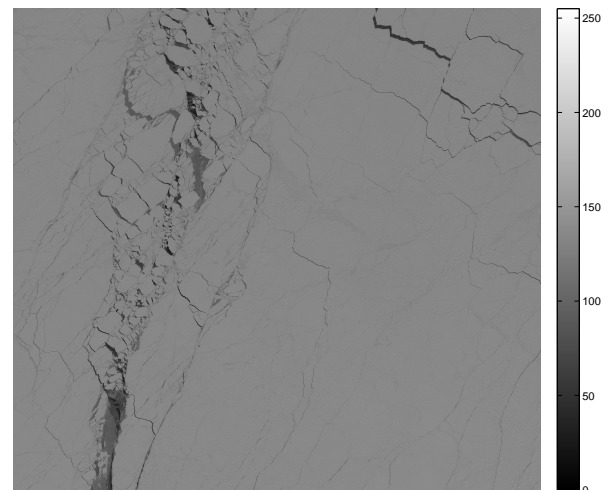

(a) Greyscale SPOT image

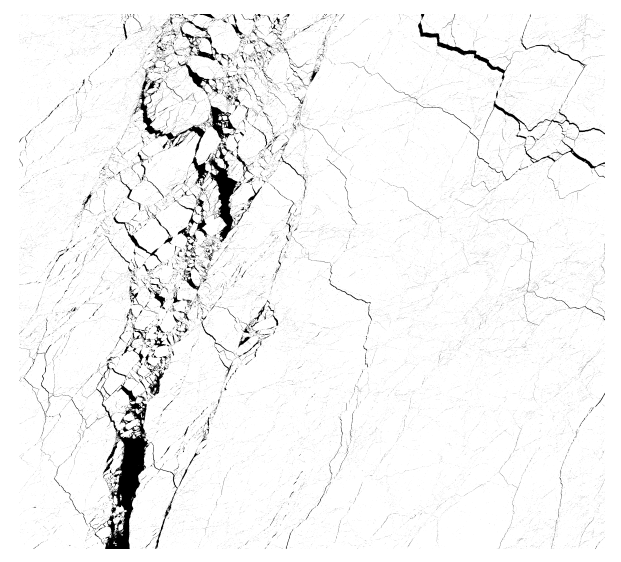

(c) $\mathrm{Th}=115$

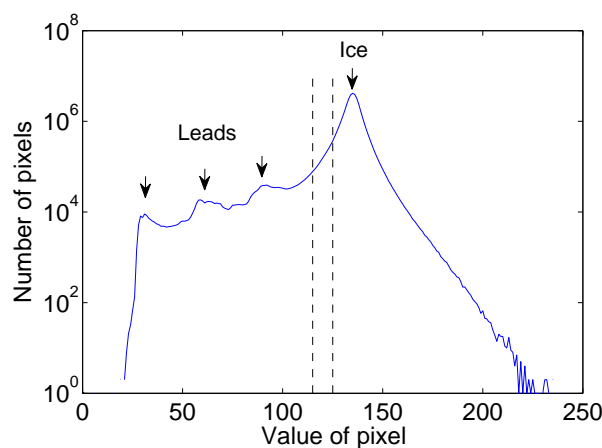

(b) Histogram of the SPOT image

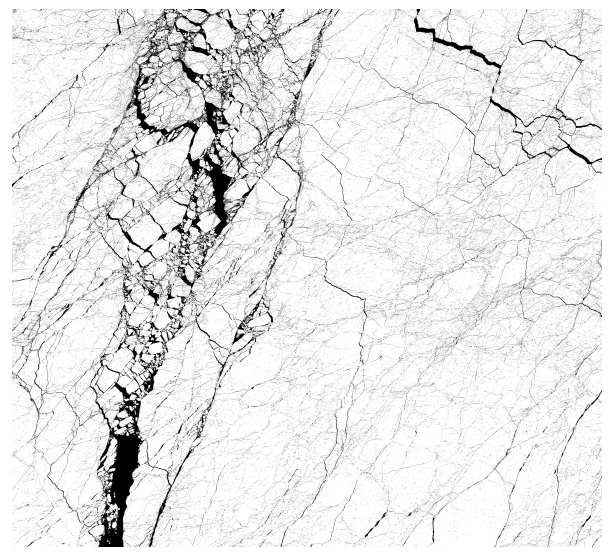

(d) $\mathrm{Th}=125$

Fig. 3. (a) Greyscale satellite SPOT image of the sea ice cover of size $5977 \times 6618$ pixels taken 6 April 1996 (i.e. early spring), centered around $80^{\circ} 11^{\prime} \mathrm{N}, 108^{\circ} 33^{\prime} \mathrm{W}$ covering $60 \times 66 \mathrm{~km}^{2}$. On the right is the luminance scale of the picture. (b) Pixel color histogram of the SPOT image; the dashed lines correspond to the two thresholds 115 and 125. (c) and (d) Binary images for th $=115$ and th $=125$.

model of Alam and Curry (1997) depends mostly on stability theory. This way, it is more sensitive to the variations in meteorological conditions. On the other hand, the purpose of the method of Andreas and Cash (1999) is to fit data that is a function of fetch, so their method is more sensitive to variation of fetch and thus lead width, and less to background conditions.

It is interesting to note that both formulations show a strong dependence of the sensible heat flux to the lead-width - and the same is true for the latent heat flux. With this result in mind, it seems obvious that the lead-width dependence of these fluxes will impact the total heat fluxes in sea ice models or GCMs that consider simplified representations of open water distribution at the grid scale.

\section{Heat fluxes for a distribution of lead widths}

\subsection{Presentation of the case}

We have chosen to consider first a real case to compare the heat fluxes calculated using either a method that takes into account the lead-width distribution or a method that does not.

Our analysis of lead-width distribution is based on a greyscale satellite SPOT image (visible wavelengths) of the sea ice cover of size $5977 \times 6618$ pixels taken 6 April 1996 (i.e. early spring), centered around $80^{\circ} 11^{\prime} \mathrm{N}, 108^{\circ} 33^{\prime} \mathrm{W}$ covering $60 \times 66 \mathrm{~km}^{2}$ with a resolution of $L_{0}=10.0 \mathrm{~m}_{\text {pixel }}{ }^{-1}$ (Fig. 3a). As in Weiss and Marsan (2004), we convert this greyscale image to a binary one of only water or ice. The threshold is determined after analyzing the luminance distribution of the image (see Fig. 3b). The three peaks associated to leads correspond to different states of refreezing over the three largest leads. There seems to be a clear distinction between the peak associated to ice and the ones associated to leads. 


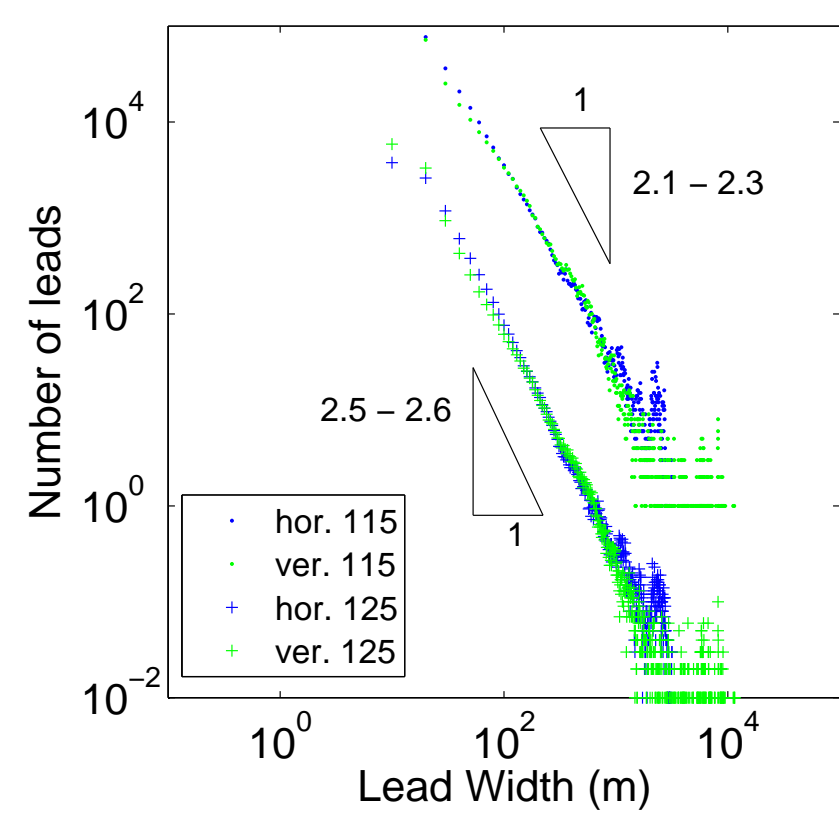

Fig. 4. Lead-width distribution along all vertical or horizontal transects on the SPOT image for the two different thresholds. We have divided the distribution associated to th $=125$ by a factor 100 for clarity purposes. The triangles are here to indicate the slope of the plots.

By converting the image to a binary one, this method does not differentiate open water from frazil or snow-free ice, which may have an impact on the value of the heat fluxes (Pinto et al., 2003). Ridges due to convergence may appear dark grey as the result of shade and therefore could be assimilated to open water although they are regions of thick ice (Weiss and Marsan, 2004). However, we assume that this does not significantly affect our results. Moreover, we will use two different luminance thresholds: 115 (Fig. 3c) and 125 (Fig. 3d) to estimate the sensitivity of the arbitrary choice of the threshold on our results.

To simulate heat fluxes, we determine lead distributions by choosing 500 random vertical or horizontal transects along Fig. 3a. Figure 4 shows that in the range $20-2000 \mathrm{~m}$, the lead-width distribution follows a power law:

$P(X)=\frac{a-1}{L_{0}}\left(\frac{X}{L_{0}}\right)^{-a}$

where $P(X)$ is the probability to have a lead of width $X$ in the transect, $a$ is the power law exponent, and $L_{0}$ is the lower bound to the power law scaling, which will be discussed later. The prefactor in this formula ensures the normalization of the probability density function: $\int_{L_{0}}^{\infty} P(X)=1$. In Sect. 3.2 and 3.3 , we will use the actual distribution of lead widths obtained for the satellite image to calculate heat fluxes. Then, in Sect. 3.4, the expression (11) will be used to test the sensitivity of the flux calculations to $a$ and $L_{0}$.
The exponents and their uncertainties (all less than $10^{-2}$ ) are calculated according to the methodology proposed by Clauset et al. (2009). Although fractures seems to have preferential directions, the power law exponent is very similar for vertical and horizontal transects: $a=2.1$ (vertical transects) and $a=2.3$ (horizontal transects) for th $=115$ and $a=2.5$ (vertical transects) and $a=2.6$ (horizontal transects) for th $=125$. Lindsay and Rothrock (1995), using radiometer (AVHRR) images, also determined that the number of leads of width $X$ was following a power law, but with a different exponent: $a \sim 1.6$. However, the scale range in their study was much larger (leads of $1-50 \mathrm{~km}$, resolution $1 \mathrm{~km}$ ), and the different exponents may indicate that the lead-width distribution may not be entirely scale invariant from the meter to the tens of kilometers scales, but that there may be different regimes for lead widths below and above the kilometer scale.

The few outliers for wide $(>2000 \mathrm{~m}$ ) leads are due to what seems the largest lead on the image, and its effect will be negligible due to the repetition of 500 transects and the fact that heat fluxes are not sensitive to variations in lead width over several hundreds of meters as we have seen above. By determining heat fluxes using 500 random transects, we get rid of the spatial variability of the SPOT image and have a more generalized distribution. The agreement between vertical and horizontal distributions shows that the power law is essentially isotropic, and therefore our analysis only weakly depends on the direction of the local wind with respect to the lead pattern.

The contribution to the upward heat flux from the ice cover is very low ( $\sim 5 \mathrm{~W} \mathrm{~m}^{-2}$ or less); for clarity purposes, we only consider the mean upward heat fluxes over open water and not over ice in all our calculations. Using these 500 lead distributions, we can calculate the weighted mean heat fluxes for an average transect using formulae from Sect. 2:

$\bar{H}=\int_{L_{0}}^{\infty} H(X) P(X) d X$

where $\bar{H}$ is the weighted mean sensible or latent heat flux, $H(X)$ is the corresponding formulation of heat flux as function of lead width from Sect. 2, and $P(X)$ is the actual distribution of leads obtained from the 500 transects in the SPOT image.

We then compare these results to the turbulent fluxes calculated using the same formulae, but assuming that all the open water constitutes one large lead and the rest of the grid is covered by ice, as in climate models. This representation will be referred to as "open". We also compare them to the fluxes calculated for a single lead whose width is the mean lead width of the distribution (columns mean 115 and mean $_{125}$ ), as in Maslanik and Key (1995):

$<H>=H(<X>)$

We finally compare these results to the turbulent fluxes calculated in the CLIO model (Coupled Large-scale Ice Ocean). 
Table 1. Sensible heat fluxes over open water calculated from the different methods: using the actual distributions in the binary images corresponding to thresholds th $=115$ and th $=125$, using only the mean lead width of these distributions, and using a distribution where all open water constitutes one large lead ("open"). The heat fluxes for (th $=115)$, (th $=125),\left(\operatorname{mean}_{115}\right),\left(\operatorname{mean}_{125}\right)$ and "open" distributions are averages of 500 simulations over 500 random horizontal or vertical transects. Heat fluxes calculated in CLIO do not depend on lead-width distributions.

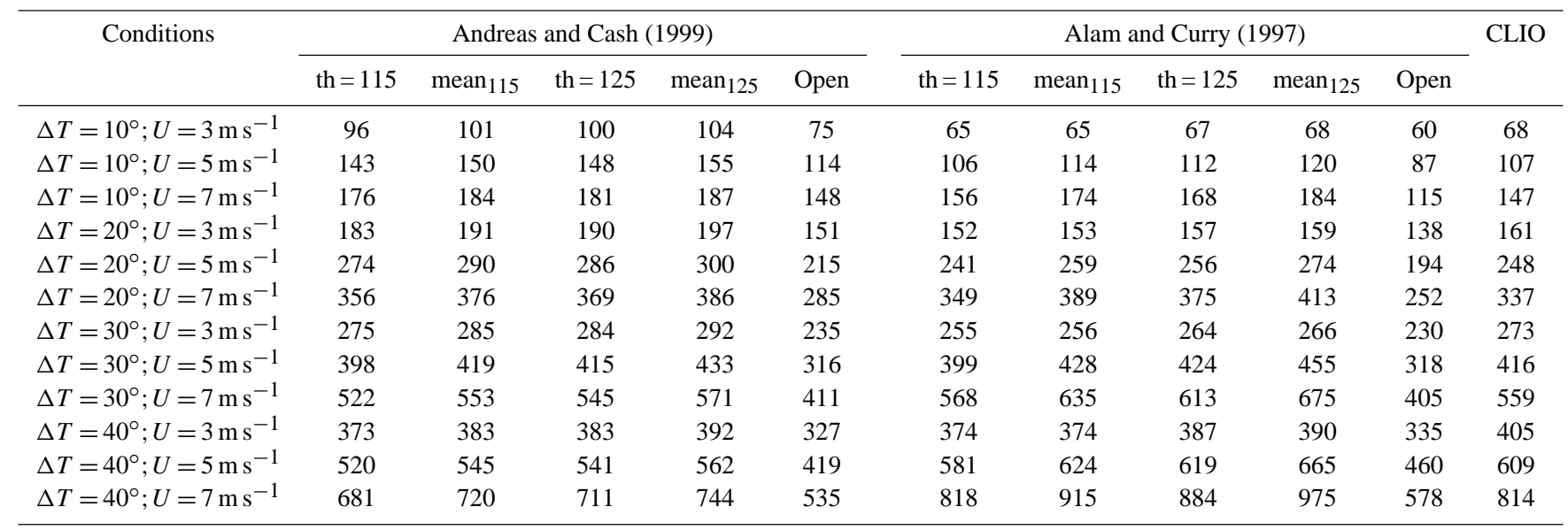

Table 2. As in Table 1 but for latent heat fluxes.

\begin{tabular}{ccccccc}
\hline Conditions & \multicolumn{5}{c}{ Andreas and Cash (1999) } & CLIO \\
\cline { 2 - 6 } & th $=115$ & mean $_{115}$ & th $=125$ & mean $_{125}$ & Open & \\
\hline$\Delta T=10^{\circ} ; U=3 \mathrm{~m} \mathrm{~s}^{-1}$ & 47 & 49 & 49 & 51 & 37 & 33 \\
$\Delta T=10^{\circ} ; U=5 \mathrm{~m} \mathrm{~s}^{-1}$ & 70 & 74 & 73 & 76 & 56 & 51 \\
$\Delta T=10^{\circ} ; U=7 \mathrm{~m} \mathrm{~s}^{-1}$ & 86 & 90 & 89 & 92 & 73 & 70 \\
$\Delta T=20^{\circ} ; U=3 \mathrm{~m} \mathrm{~s}^{-1}$ & 67 & 70 & 70 & 72 & 55 & 58 \\
$\Delta T=20^{\circ} ; U=5 \mathrm{~m} \mathrm{~s}^{-1}$ & 100 & 106 & 105 & 110 & 79 & 89 \\
$\Delta T=20^{\circ} ; U=7 \mathrm{~m} \mathrm{~s}^{-1}$ & 130 & 137 & 135 & 141 & 104 & 120 \\
$\Delta T=30^{\circ} ; U=3 \mathrm{~m} \mathrm{~s}^{-1}$ & 77 & 80 & 80 & 82 & 66 & 77 \\
$\Delta T=30^{\circ} ; U=5 \mathrm{~m} \mathrm{~s}^{-1}$ & 112 & 117 & 116 & 121 & 89 & 115 \\
$\Delta T=30^{\circ} ; U=7 \mathrm{~m} \mathrm{~s}^{-1}$ & 146 & 155 & 153 & 160 & 115 & 154 \\
$\Delta T=40^{\circ} ; U=3 \mathrm{~m} \mathrm{~s}^{-1}$ & 83 & 85 & 85 & 87 & 73 & 90 \\
$\Delta T=40^{\circ} ; U=5 \mathrm{~m} \mathrm{~s}^{-1}$ & 115 & 121 & 120 & 125 & 93 & 134 \\
$\Delta T=40^{\circ} ; U=7 \mathrm{~m} \mathrm{~s}^{-1}$ & 151 & 160 & 158 & 165 & 119 & 178 \\
\hline
\end{tabular}

It results from the coupling of a sea ice model and an ocean model both built at the Institut d'Astronomie et de Géophysique G. Lemaître, Louvain-la-Neuve (ASTR). CLIO is a state of the art model representative of coupled ocean/sea ice models used in current GCMs. Goosse et al. (2000) detail how the turbulent heat fluxes are calculated in CLIO: they have chosen to follow the work of Oberhuber (1988) that relies on widely accepted parameterizations which have a global validity. Then they have adapted their results to approach a global heat balance and to have reasonable heat transport in the ocean. Their calculation does not take into account the distribution of lead widths in their grid, and is representative of what is used in current GCMs (see Appendix B).

\subsection{Influence of lead width on mean heat fluxes}

Tables 1 and 2 show the sensible and latent heat fluxes calculated from the different methods, for different conditions of wind speed and temperature difference typical of the Arctic winter, and assuming different distributions of lead width.

For all conditions of wind and temperature, we can see that the two distributions due to the different thresholds (115 or $125)$ give very similar results, with differences less than $4 \%$ (using the method of Andreas and Cash, 1999) and less than $8 \%$ (using the method of Alam and Curry, 1997). The heat fluxes calculated for the $(\mathrm{th}=125)$ distribution are slightly larger than those for $(\mathrm{th}=115)$. As we have seen in the previous section, the exponent of the power law distribution is 
larger for $($ th $=125)$ therefore the smaller leads are even more numerous in this case than for $(\mathrm{th}=115)$, and because they produce larger heat fluxes, the overall heat fluxes are larger. We can also see that the fluxes calculated from a real distribution of leads are larger than those calculated assuming the "open" representation. This difference is about 25-40\% for the fluxes using the method of Andreas and Cash (1999) and is relatively independent of the background conditions.

On the other hand, the effect of the distribution on the method of Alam and Curry (1997) has a different influence depending on the background conditions, and especially the wind speed. The heat fluxes calculated using the method of Alam and Curry (1997) for a real distribution are 13-15\% larger than for an "open" representation for weak winds $\left(U=3 \mathrm{~m} \mathrm{~s}^{-1}\right)$, but are 45-55\% larger for strong winds $\left(U=7 \mathrm{~m} \mathrm{~s}^{-1}\right)$.

As expected, the sensible heat fluxes calculated using the method of Andreas and Cash (1999) are less sensitive to the change of background conditions. They are larger than the fluxes calculated using the method of Alam and Curry (1997) for weak wind and temperature difference (sensible heat flux of 96-100 (Andreas and Cash, 1999) and 65-67 $\mathrm{W} \mathrm{m}^{-2}$ (Alam and Curry, 1997) for $\Delta T=10^{\circ} \mathrm{C}$ and $U=3 \mathrm{~m} \mathrm{~s}^{-1}$ ) and smaller for strong wind and temperature difference (sensible heat flux of 681-711 (Andreas and Cash, 1999) and 819-885 $\mathrm{W} \mathrm{m}^{-2}$ (Alam and Curry, 1997) for $\Delta T=40^{\circ} \mathrm{C}$ and $U=7 \mathrm{~m} \mathrm{~s}^{-1}$ ).

If we compare the heat fluxes calculated from the actual distributions $(\bar{H})$ and the heat fluxes from only the mean lead width, i.e. using Eq. (13) $(<H>)$, we find that the results are comparable: there is about a $2-5 \%$ difference for the fluxes calculated with the method of Andreas and Cash (1999) and a difference of 0-12\% the fluxes calculated with the method of Alam and Curry (1997). This difference is not affected much by the change of background atmospheric conditions for the fluxes calculated with the method of Andreas and Cash (1999), but increases from no difference for low wind speed $\left(U=3 \mathrm{~m} \mathrm{~s}^{-1}\right)$ to $10-12 \%$ for high wind speed $\left(U=7 \mathrm{~m} \mathrm{~s}^{-1}\right)$, for all temperature differences between the surface and the atmosphere.

However, we believe that this relatively good agreement may be fortuitous. Indeed, the nature of the distribution is very important to determine whether the mean flux for a whole distribution is equal to the estimate of the flux for the mean lead width of the distribution. Indeed, for a power law distribution (Eq. 11) with $a>1$, the mean lead width is determined by:

$$
<X>=\int_{L_{0}}^{\infty} X P(X) d X=\frac{a-1}{L_{0}^{1-a}}\left[\frac{X^{2-a}}{2-a}\right]_{L_{0}}^{\infty}
$$

whereas the mean lead width $\langle X>$ for an exponential distribution function $P(X)=\frac{1}{\lambda} \exp \left(-\frac{X}{\lambda}\right)$ considered by Maslanik and Key (1995) is $\langle X>=\lambda$. Therefore, the mean lead width depends strongly on the exponent of the power law $a$ (and also on the lower bound $L_{0}$ as we will discuss later):

If $a<2$ (and $a>1$ ), $<X>\rightarrow \infty$ : the mean lead width is not defined because the extreme values of $X$ have too much weight. This is the case for the study of Lindsay and Rothrock (1995) who found $a=1.6$. For a real finite distribution, the mean lead width would be very large and calculating the mean heat flux using Eq. (13) as suggested in Maslanik and Key (1995) would be comparable to the calculation using the "open" distribution: this method would lead to a large under-estimation of the actual heat fluxes.

If $a>2,<X>=\frac{a-1}{a-2} L_{0}$ : the mean lead width depends on how close $a$ is to the threshold value 2, and on the lower bound $L_{0}$, and the calculation of the mean heat flux using Eq. (13) will depend on these two parameters. In Table 1 and 2, we can see that the calculation using the mean lead width for the distribution threshold $115\left(\right.$ mean $\left._{115}\right)$ is larger than the calculation using the mean lead width for the distribution threshold $125\left(\operatorname{mean}_{125}\right)$ This is because for the 115 threshold, $a=2.1-2.3$ is smaller than for the 125 threshold, $a=2.5-2.6$. Therefore, the mean lead width is larger for the distribution threshold 115, and therefore the corresponding heat flux is smaller (Fig. 2). We will see in Sect. 3.4 that this method also depends strongly on the lower bound to power law scaling $L_{0}$, which was imposed in our analysis by the resolution of the SPOT image $(10 \mathrm{~m})$, thus questioning the pertinence of an approach based on the mean lead width.

\subsection{Comparison to an ocean model}

We can see that our calculations using the method of Alam and Curry (1997) and CLIO's formulations find very similar fluxes. Even if both formulations use Monin-Obukhov similarity theory and therefore are as sensitive to the background conditions, it is surprising that they find the same results. Indeed, our calculations take into account the lead distribution - and in the previous section, we saw that the heat flux dependence on lead-width distribution was quite important and CLIO does not. The agreement between our calculations using the method of Andreas and Cash (1999) and CLIO's methods is poor and has the same reasons as explained earlier: the fluxes calculated using the method of Andreas and Cash (1999) are not as sensitive to the background conditions and increase less with instability. Overall, the heat fluxes calculated from the CLIO parameterization are in better agreement with our calculations taking into account the lead distribution than our previous calculations assuming the "open" representation. This may be due to an adjustment of the CLIO parameterizations to have reasonable values for heat fluxes. We will see in the next section that this apparent agreement may be partly fortuitous. Indeed, our calculations heavily depend on the distribution of lead widths, and particularly on the lower bound to power law scaling, which was imposed in our analysis by the resolution of the SPOT image $(10 \mathrm{~m})$. 


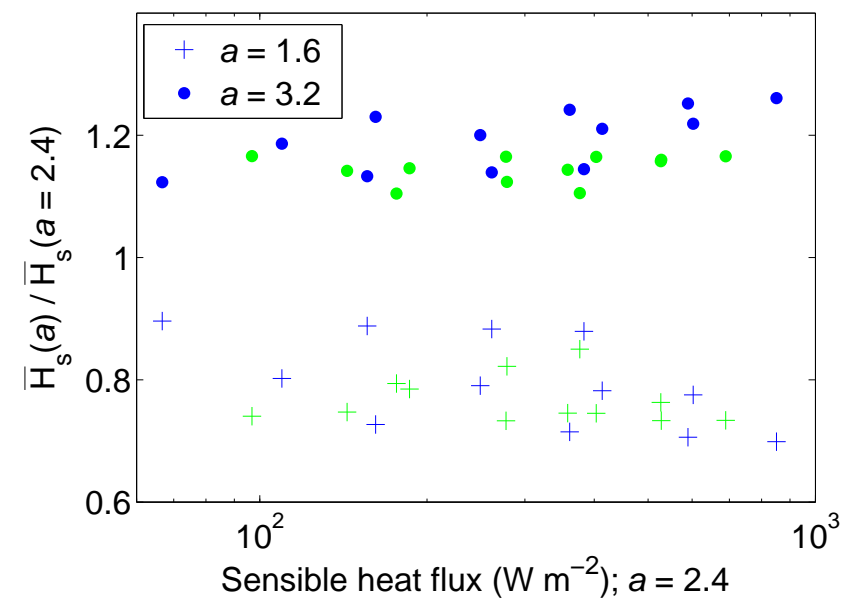

Fig. 5. Ratios of the sensible heat fluxes calculated using the methods of Andreas and Cash (1999) - green - and of Alam and Curry (1997) - blue - for two distributions of lead width exponents: $a=1.6$ (plus) and $a=3.2$ (dots) to the fluxes calculated for $a=2.4$, for the same atmospheric conditions as in Table 1.

\subsection{Sensitivity to the distribution}

We have seen in the previous section that the lead distribution in our case study follows a power law $P(X) \sim X^{-a}$ with $2.1 \leq a \leq 2.6$ for leads wider than $20 \mathrm{~m}$, a lower bound imposed by the resolution $L_{0}=10 \mathrm{~m}$ of the SPOT image. Therefore, we expect this cutoff to have little physical significance. We are not aware of other lead width statistics at this resolution. We have seen earlier that Lindsay and Rothrock (1995) have found a power law $P(X) \sim X^{-a}$ with $a=1.60 \pm 0.18$, but their resolution is $1 \mathrm{~km}$. This could mean either that the lead-width distribution is not strictly scale invariant from the tens of meters to the tens of kilometers, or that some variability on the exponent $a$ might exist. More analysis at various resolutions would be needed to answer this question.

To test the sensitivity of our flux calculations to the distribution of lead widths, we have created artificial distributions following the power law $P(X)=\frac{a-1}{L_{0}}\left(\frac{X}{L_{0}}\right)^{-a}$ by picking 50000 numbers $X=L_{0}(1-x)^{\frac{1}{1-a}}$ where $x$ is a pseudorandom number between 0 and 1 following a uniform law, and $L_{0}$ is the smallest lead resolved in our calculations, i.e. the resolution. This is equivalent to calculate the mean sensible heat flux using Eq. (12), but with the probability law defined by Eq. (11). We will first consider $L_{0}=10 \mathrm{~m}$, as imposed by the resolution of the SPOT satellite image.

First, we have tested the sensitivity of the results to a change of $35 \%$ in the exponent $a$ of the power law: from 1.6 to 3.2 (Fig. 5) and for a wide range of background atmospheric conditions. Doing so, we are simulating a leadwidth distribution with an exponent comparable to the one found by Lindsay and Rothrock (1995). We have also sim-

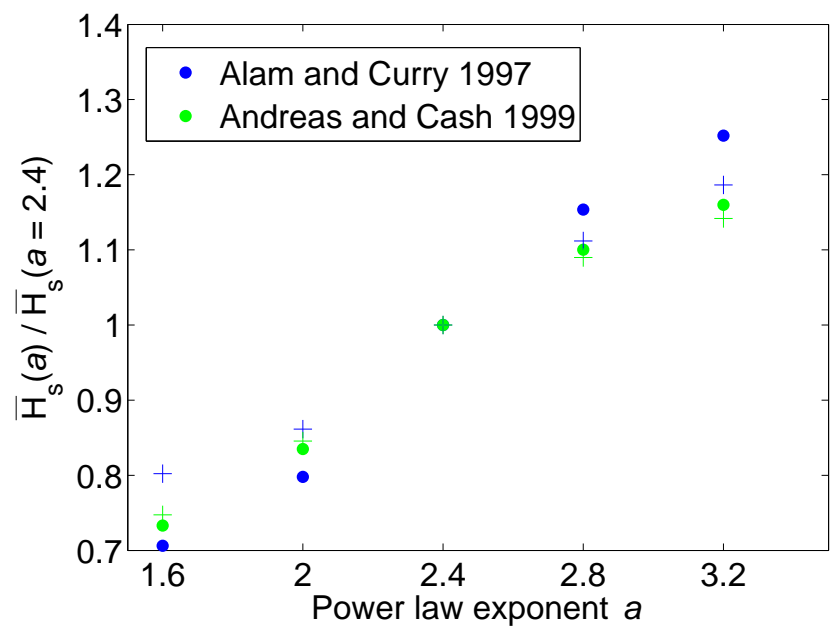

Fig. 6. Ratios of the sensible heat fluxes calculated using the methods of Andreas and Cash (1999) - green - and of Alam and Curry (1997) - blue - for several distributions of lead width exponents $a$ to the fluxes calculated for $a=2.4$, as a function of $a$ for two atmospheric conditions: $\Delta T=30^{\circ} \mathrm{C}, U=7 \mathrm{~m} \mathrm{~s}^{-1}$ (dots) and $\Delta T=10^{\circ} \mathrm{C}, U=5 \mathrm{~m} \mathrm{~s}^{-1}$ (plus).

ulated the sensitivity of the results to a change in the exponent $a$ of the power law for 2 different atmospheric conditions: $\Delta T=10^{\circ} \mathrm{C}$ and $U=5 \mathrm{~m} \mathrm{~s}^{-1}$ (plus) $\Delta T=30^{\circ} \mathrm{C}$ and $U=7 \mathrm{~m} \mathrm{~s}^{-1}$ (dots) (Fig. 6).

When $a$ increases, the distribution is shifted towards the narrow leads, which are more efficient at transferring turbulent heat, therefore $\frac{\overline{H_{\mathrm{S}}}(a>2.4)}{\overline{H_{\mathrm{S}}}(a=2.4)}>1$. And similarly $\frac{\overline{H_{\mathrm{S}}}(a<2.4)}{\overline{H_{\mathrm{S}}}}<1$.

We can see that, for a lower bound $L_{0}=10 \mathrm{~m}$, the heat fluxes are somewhat sensitive to a change in $a$. A change of $\pm 35 \%$ in the exponent $a$ results in a change of about $\pm 25 \%$ in the heat fluxes. Moreover, this difference only weakly depends on the background conditions, with a similar ratio for all ranges of fluxes. We can also notice that for large $a$, heat fluxes calculated using the method of Andreas and Cash (1999) seem less affected by the change in $a$ than those calculated using the method of Alam and Curry (1997). This is because its sensitivity to the lead width is stronger: as shown in Fig. 2, significant variations in heat flux only occur for very narrow leads $(1-25 \mathrm{~m})$. The original distribution $(a=2.4)$ is already very narrow and the resolution being equal to $10 \mathrm{~m}$, almost all leads are already in that range. This explains the small difference with fluxes calculated for even narrower distributions, i.e. $a>2.4$. Overall, we can say that for a lower bound of $10 \mathrm{~m}$, the sensitivity of our calculations to the exponent $a$ is relatively small, which supports our approach of using the distribution found in only one particular SPOT image, even if this distribution is not representative of all sea ice. 


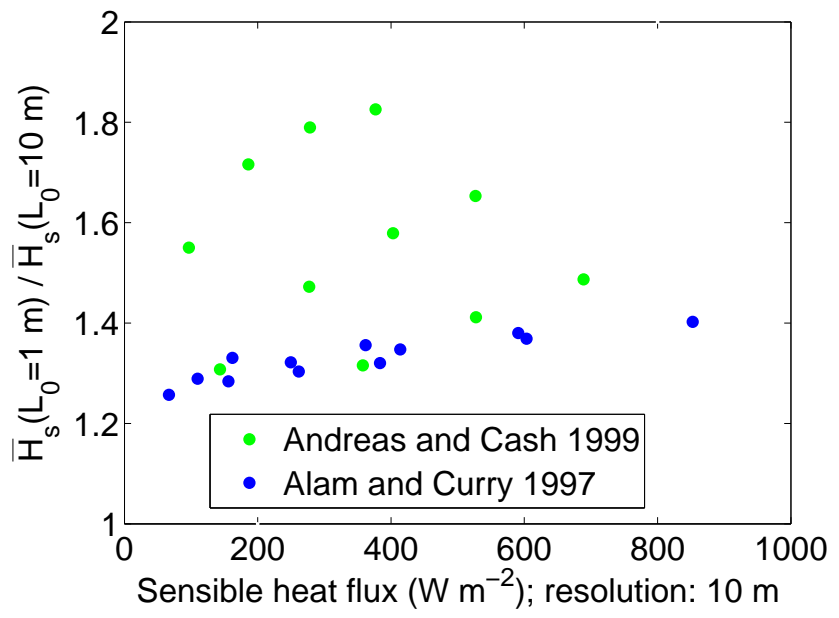

Fig. 7. Ratio of the sensible heat fluxes calculated with resolution $L_{0}=1 \mathrm{~m}$ to $L_{0}=10 \mathrm{~m}$ using the methods of Andreas and Cash (1999) - green - and of Alam and Curry (1997) - blue - for the same power law exponent $a=2.4$ and for the same atmospheric conditions as in Table 1 . Note that in this calculation, the four points in the upper left corner are obtained using the method of Andreas and Cash (1999) are for low wind speed $\left(U=3 \mathrm{~m} \mathrm{~s}^{-1}\right)$.

We have also tested the sensitivity of the results to the smallest lead that can be resolved, i.e. to $L_{0}$, the lower bound to power law scaling on Fig. 4. Unlike the exponent $a$, this parameter does not have a physical meaning and depends on the resolution of the SPOT image. The probability distribution depends significantly on this variable: if the lead width follows a power law distribution down to scales smaller than $10 \mathrm{~m}$ with exponent $a=2.4$, leads smaller than $10 \mathrm{~m}$ would be even more abundant, e.g. there would be about $\left(\frac{5}{10}\right)^{-a} \sim 5$ times more $5 \mathrm{~m}$-wide leads than $10 \mathrm{~m}$ wide leads. As the heat fluxes vary significantly with lead width at this scale, the overall influence should be important. We have chosen to consider a minimal lead width $L_{0}=1 \mathrm{~m}$, which is the order of magnitude of sea ice thickness. Alam and Curry (1997) have calculated heat fluxes down to $1 \mathrm{~m}$ and Andreas and Cash (1999) do not give a range of validity for their formulations but they have used data down to fetches of several meters, and so we will assume that their formulations will give a reasonable estimate at a resolution of $1 \mathrm{~m}$.

As expected, both methods are sensitive to the smallest lead width that can be resolved (Fig. 7). Changing the smallest lead considered in our calculation leads to an increase of the heat fluxes of $20-40 \%$ using the method of Alam and Curry (1997) and of 20-180\% using the method of Andreas and Cash (1999). We can see that the sensitivity of the fluxes to the lower bound $L_{0}$ seems to increase with the heat flux, i.e. with the background instability. The large change obtained using the method of Andreas and Cash (1999) is due to the fact the formulation is the most sensitive to lead width for narrow leads, as seen in previous sections. This method's important sensitivity at small scales may explain the large variability of results for different background conditions. An other explanation may be that in these conditions (especially for low wind speeds $-3 \mathrm{~m} \mathrm{~s}^{-1}$ - where the increase is the most important), there is a change of regime with mostly forced convection for scales of $1 \mathrm{~m}$ and mostly free convection for scales of $10 \mathrm{~m}$ and so very different heat fluxes.

The role of the lower bound $L_{0}$ is important because it shows that the resolution of the SPOT image has a strong influence on the calculation of the turbulent heat fluxes, and probably in the good agreement between the values of heat fluxes calculated using the lead-width distribution obtained from the SPOT image, and CLIO, which is representative of large models and GCMs (Table 1). In other words, this agreement may be partly fortuitous.

The same is true for a parameterization based on the mean lead width only: if we calculate the heat flux using Eq. (13) with a mean lead width $\langle X\rangle=\frac{a-1}{a-2} L_{0}$ for e.g. $a=2.4$ and $L_{0}=1 \mathrm{~m}$ with the method of Andreas and Cash (1999), then $<X>=3.5 \mathrm{~m}$ and $\overline{H_{\mathrm{s}}}=153 \mathrm{~W} \mathrm{~m}^{-2}$ for $\Delta T=10^{\circ} \mathrm{C}, U=$ $3 \mathrm{~m} \mathrm{~s}^{-1}$ and $\overline{H_{\mathrm{s}}}=790 \mathrm{~W} \mathrm{~m}{ }^{-2}$ for $\Delta T=30^{\circ} \mathrm{C}, U=7 \mathrm{~m} \mathrm{~s}^{-1}$, i.e. in both cases an overestimation of about $50 \%$ of the heat fluxes. Therefore, the correct agreement seen in Sect. 3.2 between the calculations using the full lead-width distributions and the calculations using only the mean lead width is most likely fortuitous.

This proves that the distribution of lead width is very important, especially at small scales $(1-10 \mathrm{~m})$, because it is at these scales that it has the strongest influence on heat flux calculations. This argues for further observations and analyses at such small scales.

\section{Discussion}

In our calculations, we have only considered the leads individually. However, the spatial distribution of leads is likely to play a role in the turbulent heat transfers. In a modeling study, Lüpkes et al. (2008a) showed that the convective plume created by a $1 \mathrm{~km}$-wide lead extended over several kilometers downwind of the lead and could therefore affect leads in that area. Moreover, the moisture released by water openings in a cold environment favors the apparition of clouds: Khvorostyanov et al. (2003) have combined data and a model to simulate the cloud created over the Beaufort Sea polynya (about $20 \mathrm{~km}$ wide). Such a cloud extended for more than $100 \mathrm{~km}$ downwind and played a large role in the radiative balance of the area. Therefore, the effect of leads on each other through heating due to the release of sensible heat or through the formation of clouds should be investigated in more detail.

Our formulations taking into account the distribution of lead widths are based on binary images where the surface is either open water or thick ice. However, the histogram of 
the SPOT image (Fig. 3b) shows that the peaks associated to leads correspond to different colors. This is due to the fact that there are different states of refreezing over leads. Pinto et al. (2003) have studied the refreezing of leads and noticed that the surface of the openings wider than $20 \mathrm{~m}$ was refrozen after 5-24 $\mathrm{h}$. The refreezing process has different stages and the heat fluxes over a lead decrease as newly-formed ice gets thicker. Ultimately, the heat fluxes over a refrozen lead are equal to their minimal values once the ice is covered by snow.

Moreover, Lüpkes et al. (2008b) described that the upward heat transport over leads results in a corresponding downward heat transport over nearby sea ice. This means that the dependence of upward heat flux on lead width would generate also a width dependence of the downward flux and stability over the ice surface. Therefore, the lead-width distribution would also affect the nearby sea-ice and may have major impact on its structure and characteristics.

\section{Conclusions}

Turbulent heat fluxes over sea ice leads depend strongly on the lead width and decrease with increasing lead width. Narrow leads (several meters) are over two times more efficient at transmitting turbulent heat than larger ones (several hundreds of meters). Leads over several hundreds meters can be considered as open ocean and heat fluxes over them do not depend on lead width anymore.

Using a SPOT satellite image of Arctic sea ice, we have seen that the distribution of lead width follows a power law $P(X) \sim X^{-a}$ with $a \sim 2.4$, for leads of widths $20-2000 \mathrm{~m}$. Narrow leads are therefore the most abundant.

We have found that the mean heat fluxes over open water are up to $55 \%$ larger when considering the lead-width distribution obtained from the SPOT image, compared to the situation where the open water is concentrated in one large lead. Therefore the lead-width distribution should be taken into account when calculating heat fluxes over open water.

Climate model parameterizations do not take into account the lead-width distribution explicitly. We have compared our calculations of heat fluxes that take into account the leadwidth distribution with heat fluxes as calculated by a state of the art coupled ocean/sea ice model (CLIO). Although CLIO does not take into account the lead-width distribution, the difference with our estimations is somewhat reduced (5-30\%). However, this relatively good agreement may be partly fortuitous. Indeed, the heat fluxes calculated for a power law distribution of lead widths heavily depends on the lower bound of scale invariance. In the case analyzed here, this bound was imposed by the resolution of the image $(10 \mathrm{~m})$. If scaling holds down to smaller scales (e.g. $1 \mathrm{~m}$ ), calculated heat fluxes would increase by up to a factor of 2 , and the agreement with CLIO will no longer hold. Similarly, a parameterization of heat fluxes based only on the mean lead width $<X>$, which is directly dependent on the lower bound to scaling for a power law distribution with $a>2$, is most likely not appropriate.

This work shows that turbulent heat transfers between the ocean and the atmosphere in ice-covered oceans strongly depends on the distribution of lead widths, especially at very small scales (smaller than $50 \mathrm{~m}$ ). Consequently, a better characterization of lead patterns at such fine scales is worth pursuing. Our estimations may be a first step towards a subgrid scale parameterization of the spatial distribution of open water for heat flux calculations in ocean/sea ice coupled models.

\section{Appendix A}

\section{Equations from Andreas and Cash (1999)}

In this Appendix are the equations from Andreas and Cash (1999) that were used in this study. The reader is advised to consult the original article for more details. Some symbols that are not defined here are defined in Appendix C.

$$
\begin{aligned}
& H_{\mathrm{S}}(X)=C_{*} \rho c_{\mathrm{p}} D \frac{\Delta T}{\Delta z_{T}} \\
& H_{\mathrm{l}}(X)=C_{*} \rho L_{\mathrm{v}} D_{\mathrm{w}} \frac{\Delta Q}{\Delta z_{Q}}
\end{aligned}
$$

where $H_{\mathrm{s}}$ and $H_{1}$ are the upward fluxes of sensible and latent heat, respectively, as a function of the lead width $X, \Delta z_{T}$ and $\Delta z_{Q}$ are two length scales for heat and humidity, respectively, that take into account the viscosity of air and the buoyancy difference between the surface $s$ and the altitude $r$, $\rho$ is the air density, $c_{\mathrm{p}}$ is the specific heat of air at constant pressure, $L_{\mathrm{v}}$ is the latent heat of evaporation, $D$ and $D_{\mathrm{w}}$ are the molecular diffusivities of heat and water vapor in air, respectively, and $\Delta T=T_{s}-T_{r}, \Delta Q=Q_{s}-Q_{r}$, changes in heat and humidity, respectively between surface $s$ and altitude $r$. Note that the altitude $r$ corresponds to the first level of measurements in the atmosphere.

The empirical non-dimensional coefficient $C_{*}$ has been determined as a function of stability from the lead and polynya data:

$$
C_{*}=\frac{0.3}{0.4-h / L}+0.15
$$

where $h$ is the TIBL depth in meters as a function of lead width $X$ :

$h=0.82 \ln (X)+0.02$

and $L$ is the Obukhov length, defined later in this appendice. This formulation of the TIBL depth comes from lead and polynyas data (Fig. 1 of Andreas and Cash, 1999).

$\Delta z_{T}$ and $\Delta z_{Q}$ are defined by:

$\Delta z_{T}=\left(\frac{\nu D}{\Delta B}\right)^{1 / 3}$ 
$\Delta z_{Q}=\left(\frac{\nu D_{\mathrm{w}}}{\Delta B}\right)^{1 / 3}$

where $v$ is the kinematic viscosity of air and $\Delta B$ is the buoyancy difference:

$\Delta B=\frac{g}{\bar{T}}\left(\Delta T+\frac{0.61 \bar{T} \Delta Q}{1+0.61 \bar{Q}}\right)$

where $\bar{T}=\frac{1}{2}\left(T_{r}+T_{s}\right)$ and $\bar{Q}=\frac{1}{2}\left(Q_{r}+Q_{s}\right)$ are the mean temperature and humidity, respectively, between the surface $s$ and altitude $r$.

$L$ is the Obukhov length, calculated using the method defined in Andreas and Murphy (1986):

$L^{-1}=8.0\left(\frac{0.65}{r}+0.079-0.0043 r\right) R i_{\mathrm{b}}$

$R i_{\mathrm{b}}=-\frac{r g}{\bar{T}} \frac{T_{s}-T_{r}}{U_{r}^{2}}$

\section{Appendix B}

\section{Equations to compute heat fluxes as in CLIO}

We have followed the method described in Goosse et al. (2000), to calculate heat fluxes over open water in unstable conditions. The reader is advised to consult the original article for more details.

$H_{\mathrm{s}}=\rho c_{\mathrm{p}} c_{\mathrm{sh}} U_{r}\left(T_{s}-T_{r}\right)$

$H_{1}=\rho L_{\mathrm{w}} c_{\mathrm{le}} U_{r}\left(q_{s}-q_{r}\right)$

where $U_{r}, T_{r}$ and $q_{r}$ are the air velocity, temperature and specific humidity, respectively, at the altitude $r=10 \mathrm{~m}$. $T_{s}$ and $q_{s}$ are the temperature and specific humidity right at the surface of the open water. $q_{s}$ is calculated as a function of the vapor pressure at saturation $e_{s}$ (in $\mathrm{Pa}$ ):

$q_{s}=\frac{0.622 e_{s}}{P-0.378 e_{s}}$

$e_{S}=611 \times 10^{\left(T_{r}-273.16\right) /\left(T_{r}-b\right)}$

where $P=10^{5} \mathrm{~Pa}$ is the atmospheric pressure, $(a, b)=$ $(7.5,35.86)$ are two empirical coefficients.

The transfer coefficients $c_{\mathrm{sh}}$ and $c_{\mathrm{le}}$ are:

$$
\begin{aligned}
c_{\mathrm{sh}} & =0.0327 \frac{k}{\ln \left(r / z_{0}\right)} \Phi_{\mathrm{sh}}\left(T_{r}, T_{s}, q_{r}, q_{s}, u_{*}\right) \\
& =c_{\mathrm{shN}} \Phi_{\mathrm{sh}}\left(T_{r}, T_{s}, q_{r}, q_{s}, u_{*}\right) \\
c_{\mathrm{le}} & =0.0346 \frac{k}{\ln \left(r / z_{0}\right)} \Phi_{\mathrm{le}}\left(T_{r}, T_{s}, q_{r}, q_{s}, u_{*}\right) \\
& =c_{\mathrm{leN}} \Phi_{\mathrm{le}}\left(T_{r}, T_{s}, q_{r}, q_{s}, u_{*}\right)
\end{aligned}
$$

with $z_{0}=0.032 \frac{u_{*}^{2}}{g}$ is the roughness length, $u_{*}$ is the friction velocity. $c_{\mathrm{shN}}$ and $c_{\mathrm{leN}}$ are the expression of the transfer coefficients $c_{\mathrm{sh}}$ and $c_{\text {le }}$ for neutral conditions.

$\Phi_{\mathrm{sh}}$ and $\Phi_{\mathrm{le}}$ are functions that take into account the stability of the air above the surface. In stable conditions, they are equal to 1 and in unstable conditions,

$$
\begin{aligned}
& \Phi_{\mathrm{sh}}\left(T_{r}, T_{s}, q_{r}, q_{s}, u_{*}\right)=\frac{\sqrt{c_{\mathrm{M}} / c_{\mathrm{MN}}}}{1-c_{\mathrm{shN}} k^{-1} C_{\mathrm{MN}}^{-1 / 2} \Psi_{H}(r / L)} \\
& \Phi_{\mathrm{le}}\left(T_{r}, T_{s}, q_{r}, q_{s}, u_{*}\right)=\frac{\sqrt{c_{\mathrm{M}} / c_{\mathrm{MN}}}}{1-c_{\mathrm{leN}} k^{-1} C_{\mathrm{MN}}^{-1 / 2} \Psi_{\mathrm{L}}(r / L)}
\end{aligned}
$$

where the coefficients $c_{\mathrm{M}}, c_{\mathrm{MN}}$ are defined by:

$$
\begin{aligned}
& \sqrt{c_{\mathrm{M}} / c_{\mathrm{MN}}}=\left(1-\sqrt{C_{\mathrm{MN}}} k^{-1} \Psi_{\mathrm{M}}(r / L)\right)^{-1} \\
& c_{\mathrm{MN}}=\frac{k^{2}}{\ln ^{2}\left(r / z_{0}\right)}
\end{aligned}
$$

where $k=0.4$ is the von Karman constant,

$$
T_{0}=T_{r}\left(1+2.2 \times 10^{-3} T_{r} q_{r}\right)
$$

where $\Psi_{\mathrm{M}}, \Psi_{\mathrm{H}}$ and $\Psi_{\mathrm{L}}$ are the stability functions for momentum, heat and moisture, respectively:

$$
\begin{aligned}
& \Psi_{\mathrm{M}}(A)=2 \ln \left(\frac{1+A}{2}\right)+\ln \left(\frac{1+A^{2}}{2}\right)-2 \arctan A+\pi / 2 \\
& \Psi_{\mathrm{H}}(A)=\Psi_{\mathrm{L}}(A)=2 \ln \left(\frac{1+A^{2}}{2}\right)
\end{aligned}
$$

with $A=(1-16(r / L))^{1 / 4}$

$r / L=\frac{100 r}{T_{0} u_{*}^{2} / c_{\mathrm{M}}}\left(\left(T_{r}-T_{s}+2.2 \times 10^{-3} T_{0}^{2}\left(q_{s}-q_{r}\right)\right)\right.$

\section{Appendix C}

\section{Constants}

$\rho$ : the air density is computed from the equation of state of the perfect gases.

$c_{\mathrm{p}}=1005 \mathrm{~J} \mathrm{Kg}^{-1} \mathrm{~K}^{-1}$ : specific heat of air.

$L_{\mathrm{w}}=2.5 \times 10^{-6} \mathrm{~J} \mathrm{Kg}^{-1}$ the latent heat of evaporation.

$g=9.8 \mathrm{~m} \mathrm{~s}^{-2}$ : acceleration due to gravity.

$D=1.86 \times 10^{-5} \mathrm{~m}^{2} \mathrm{~s}^{-1}$ : molecular diffusivity of heat in the air.

$D_{\mathrm{w}}=2.14 \times 10^{-5} \mathrm{~m}^{2} \mathrm{~s}^{-1}$ : molecular diffusivity of water vapor in the air.

$v=1.31 \times 10^{-5} \mathrm{~m}^{2} \mathrm{~s}^{-1}$ : kinematic viscosity of air.

$k=0.4$ : von Karman constant. 
Acknowledgements. This work has been supported by CNRS. We thank H. Gallée for stimulating discussions, as well as E. Andreas, C. Lüpkes and M. Vancoppenolle for valuable comments on the manuscript.

Edited by: M. Van den Broeke

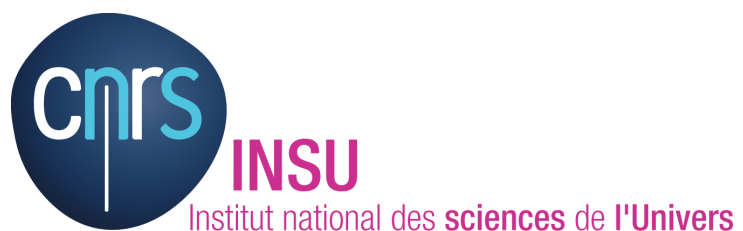

The publication of this article is financed by CNRS-INSU.

\section{References}

Alam, A. and Curry, J.: Determination of surface turbulent fluxes over leads in Arctic sea ice, J. Geophys. Res., 102, 3331-3343, 1997.

Andreas, E. and Cash, B.: Convective heat transfer over wintertime leads and polynyas, J. Geophys. Res., 104, 25721-25734, 1999.

Andreas, E. and Murphy, B.: Bulk transfer coefficients for heat and momentum over leads and polynyas, J. Phys. Oceanogr., 16, 1875-1883, 1986.

Andreas, E., Paulson, C., William, R., Lindsay, R., and Businger, J.: The turbulent heat flux from Arctic leads, Bound.-Lay. Meteorol., 17, 57-91, 1979.

Benoit, R.: On the integral of the surface layer profile-gradient functions., J. Appl. Meteorol., 16, 1977.

Bitz, C., Holland, M., Weaver, A., and Eby, M.: Simulating the icethickness distribution in a coupled climate model, J. Geophys. Res., 106, 2441-2463, 2001.

Bourassa, M., Vincent, D., and Wood, W.: A sea state parameterization with nonarbitrary wave age applicable to low and moderate wind speeds, J. Phys. Oceanogr., 31, 2840-2851, 2001.

Brutsaert, W.: A theory for local evaporation (or heat transfer) from rough and smooth surfaces at ground level, Water Resour. Res., 11, 543-550, 1975.

Clauset, A., Shalizi, C., and Newman, M.: Power-law distributions in empirical data, SIAM Rev., 51, 661-703, 2009.

Clayson, C., Fairall, C., and Curry, J. A.: Evaluation of turbulent fluxes at the ocean surface using surface renewal theory, J. Geophys. Res., 101, 28503-28513, 1996.

Fichefet, T. and Morales Maqueda, M. A.: On modelling the sea-ice - ocean system, Problemes non lineaires appliques 1994-1995, Modelisations couplees en climatologie, 76, 343-420, 1995.

Goosse, H. and Fichefet, T.: Importance of ice-ocean interactions for the global ocean circulation: A model study, J. Geophys. Res., 104, 23337-23355, 1999.

Goosse, H., Campin, J., Deleersnijder, E., Fichefet, T., Mathieu, P., Morales Maqueda, M., and Tartinville, B.: Description of the CLIO model version 3.0, Scientific Report, 3, 2000.

Gordon, H. and O'Farrell, S. P.: Transient climate change in the CSIRO coupled model with dynamic sea ice, Mon. Weather Rev., 125, 875-907, 2010.

Harvey, L.: A semianalytic energy balance climate model with explicit sea ice and snow physics, J. Climate, 1, 1065-1085, 1988.
Heil, P. and Hibler, W.: Modeling the high-frequency component of Arctic sea ice drift and deformation, J. Phys. Oceanogr., 32, 3039-3057, 2002.

Khvorostyanov, V., Curry, J., Gultepe, I., and Strawbridge, K.: A springtime cloud over the Beaufort Sea polynya: Threedimensional simulation with explicit spectral microphysics and comparison with observations, J. Geophys. Res., 108, 4296, doi:10.1029/2001JD001489, 2003.

Ledley, T.: A coupled energy balance climate-sea ice model: Impact of sea ice and leads on climate, J. Geophys. Res., 93, 1591915932, 1988.

Lindsay, R. and Rothrock, D.: Arctic sea ice leads from advanced very high resolution radiometer images, J. Geophys. Res., 100, 4533-4544, 1995.

Lüpkes, C., Gryanik, V. M., Witha, B., Gryschka, M., Raasch, S., and Gollnik, T.: Modeling convection over arctic leads with LES and a non-eddy-resolving microscale model, J. Geophys. Res., 113, C09028, doi:10.1029/2007JC004099, 2008a.

Lüpkes, C., Vihma, T., Birnbaum, G., and Wacker, U.: Influence of leads in sea ice on the temperature of the atmospheric boundary layer during polar night, Geophys. Res. Lett., 35, L03805, doi:10.1029/2007GL032461, 2008b.

Makshtas, A.: The heat budget of Arctic ice in the winter, Int. Glaciol. Soc., Cambridge, UK, 1991.

Maslanik, J. and Key, J.: On treatments of fetch and stability sensitivity in large-area estimates of sensible heat flux over sea ice, J. Geophys. Res., 100, 4573-4584, 1995.

Maykut, G.: Large-Scale Heat Exchange and Ice Production in the Central Arctic, J. Geophys. Res., 87, 7971-7984, 1982.

Maykut, G.: The surface heat and mass balance, in: The Geophysics of Sea Ice, NATO ASI Series B Phys., vol 146, edited by: Untersteiner, N., 395-463, Plenum Press, 1986.

Miles, M. and Barry, R.: A 5-year satellite climatology of winter sea ice leads in the western Arctic, J. Geophys. Res., 103, 2172321734, 1998.

Monin, A. and Obukhov, A.: Basic laws of turbulent mixing in the surface layer of the atmosphere, Contrib. Geophys. Inst. Acad. Sci., 1954.

Oberhuber, J.: An atlas based on the 'COADS' data set: The budgets of heat, buoyancy and turbulent kinetic energy at the surface of the global ocean, Max-Planck-Institut fuer Meteorologie, Rep. 15, Hamburg, Germany, 1988.

Overland, J., McNutt, S., Groves, J., Salo, S., Andreas, E., and Persson, P.: Regional sensible and radiative heat flux estimates for the winter Arctic during the Surface Heat Budget of the Arctic Ocean (SHEBA) experiment, J. Geophys. Res., 105, 1409314102, 2000.

Pinto, J., Alam, A., Maslanik, J., and Curry, J.: Surface characteristics and atmospheric footprint of springtime Arctic leads at SHEBA, J. Geophys. Res., 108, 8051, doi:10.1029/2000JC000473, 2003.

Rampal, P., Weiss, J., and Marsan, D.: Positive trend in the mean speed and deformation rate of Arctic sea ice, 1979-2007, J. Geophys. Res., 114, C05013, doi:10.1029/2008JC005066, 2009.

Smith, S., Anderson, R., Hartog, G. D., Topham, D., and Perkin, R.: An investigation of a polynya in the Canadian Archipelago 2, Structure of turbulence and sensible heat flux, J. Geophys. Res., 88, 2900-2910, 1983.

Stull, R. B.: An introduction to boundary layer meteorology, At- 
mos. Ocean. Sci. Lib., Kluwer Academic Publishers, Dordrecht, The Netherlands, 1988.

Vavrus, S.: Sensitivity of the Arctic climate to leads in a coupled atmosphere-mixed layer ocean model, J. Climate, 8, 158-171, 1995.
Venkatram, A.: A model of internal boundary-layer development, Bound.-Lay. Meteorol., 11, 419-437, 1977.

Weiss, J.: Scaling of fracture and faulting of ice on earth, Surv. Geophys., 24, 185-227, 2003.

Weiss, J. and Marsan, D.: Scale properties of sea ice deformation and fracturing, C. R. Physique, 5, 735-751, 2004. 